\title{
ARTICLE
}

\section{Neutronics design on DD neutron spectrometer for KSTAR}

\author{
Yosuke Yamamoto $^{\mathrm{a}}$, Hideki Tomita ${ }^{\mathrm{a}}$, Mitsutaka Isobe ${ }^{\mathrm{b}}$, MunSeong Cheon ${ }^{\mathrm{c}}$, Kunihiro Ogawa ${ }^{\mathrm{b}}$, Fumitaka Yamashita ${ }^{\mathrm{a}}$, \\ Jun Kawarabayashi ${ }^{\mathrm{a}}$ and Tetsuo Iguchi $^{\mathrm{a}}$ \\ ${ }^{a}$ Graduate School of Engineering, Nagoya University, Furo-cho, Chikusa-ku, Nagoya, 464-8603, Japan; ${ }^{b}$ National Institute for \\ Fusion Science, 322-6 Oroshi-cho, Toki, 509-5292, Japan; ${ }^{c}$ National Fusion Research Institute, Daejeon, 169-148, Korea
}

\begin{abstract}
Toward fusion plasma diagnostics using DD neutron emitted from deuterium plasma, we installed a DD neutron spectrometer in magnetic confinement fusion experimental device, the Korea Superconducting Tokamak for Advance Research (KSTAR) at the National Fusion Research Institute (NFRI), Korea. We developed a Monte-Carlo model of KSTAR for neutron transport calculation based on PHITS and considered a neutronics design of a shielding structure including a collimator for a prototype of a DD neutron spectrometer.
\end{abstract}

\section{Keywords: DD neutron; neutron spectrometer; nuclear fusion; neutronics design}

\section{Introduction}

Comprehensive understanding of toroidal fusion plasma is important to control a high temperature plasma in magnetic confinement fusion experimental device toward nuclear fusion energy. From deuterium fusion plasma, DD neutron with energy of around $2.5 \mathrm{MeV}$ is emitted as one of accompanying products of DD fusion reaction. In deuterium plasma heated by deuterium beam injection, emission rate of DD neutrons is normally dominated by a beam-plasma reaction. Thus, an energy spectrum of DD neutron without scattering by vacuum vessel etc. depends on velocity and density distributions of energetic deuterium ions. Deuterium plasma experiment is planned in the Large Helical Device (LHD) at the National Institute of Fusion Science (NIFS), Japan. To measure DD neutron spectra for energetic ions diagnostics in LHD, we have developed the Associated Particle Coincident Counting Neutron Spectrometer (APCCNS) as the DD neutron spectrometer [1]. In the Korea Superconducting Tokamak for Advance Research (KSTAR) at the National Fusion Research Institute (NFRI), Korea, deuterium plasma experiment started in 2010. We proposed DD neutron measurement using APCCNS in KSTAR. For installation of APPCNS in KSTAR, a Monte-Carlo model of KSTAR, i.e. an input file for neutron transport calculation in KSTAR based on Particle and Heavy Ion Transport code System (PHITS) [2] was developed. In this paper, we described a neutronics design of a shielding structure including a collimator for a prototype of APCCNS.

*Corresponding author. Email: tomita@nagoya-u.jp

\section{Detection principle of APCCNS and installation in KSTAR}

The detailed principle of DD neutron measurement and performance of the prototype of APCCNS were described in our previous paper [1]. Detection principle of APCCNS is described only briefly here. Figure 1 shows a drawing of the prototype of APCCNS. The spectrometer is based on coincident detection of a scattered neutron and a recoiled proton associated to an event of neutron elastic scattering by hydrogen in a radiator. The incident neutron energy is derived from sum of deposit energies of the recoiled proton in both the radiator and the recoiled proton detector and the scattered neutron energy measured by a time-of-flight technique. Since the incident neutron energy is independent on a scattered angle $\theta$, high energy resolution would be available. Coincident detection of

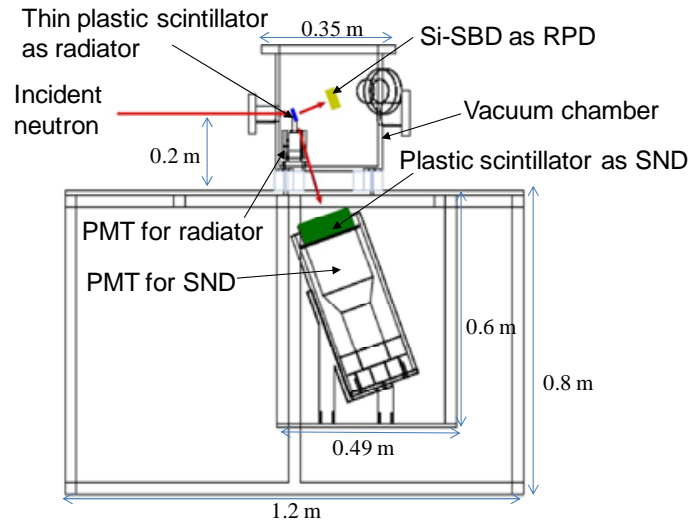

Figure 1. Drawing of the prototype of APCCNS. Si-SBD: Si Surface Barrier Detector, PMT: Photo Multiplier Tube, SND: Scattered Neutron Detector, RPD: Recoiled Proton Detector. 
the three detectors reduces accidental coincidence detections due to X-ray and gamma-ray events. For 2.5 $\mathrm{MeV}$ neutron, an energy resolution of $6.3 \%$ in FWHM and an detection efficiency of $3.3 \times 10^{-7}$ counts/neutron were demonstrated using the prototype of APCCNS [1].

Figure 2 shows a port layout of KSTAR and a position of the spectrometer. The prototype of APCCNS was installed on a deck in the front of J port of KSTAR for 2012 experimental campaign. The spectrometer has a tangential line of sight that crosses the KSTAR plasma from J port.

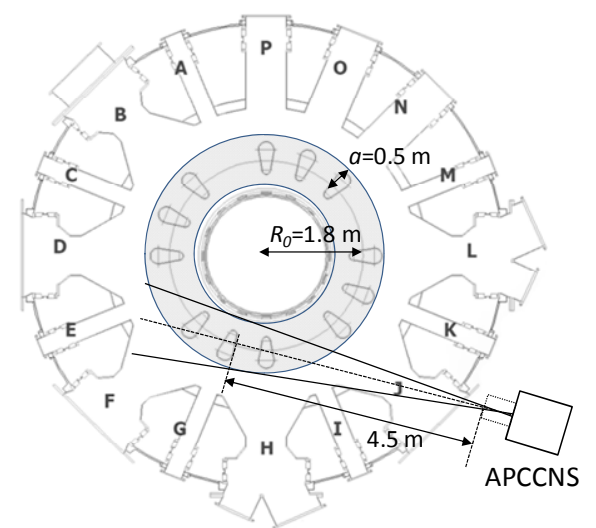

Figure 2. Port layout of KSTAR and the position of the spectrometer.

\section{Design of shielding structure}

\subsection{Monte-Carlo model of KSTAR}

A Monte-Carlo model of KSTAR was developed to calculate neutron transport for neutronics design. Figure $\mathbf{3}$ shows the Monte-Carlo model of KSTAR and material specification. Materials used in the model were as follows: vacuum vessel and cryostat were SUS316, superconductor coils were $\mathrm{Nb}_{3} \mathrm{Sn}$ and $\mathrm{Cu}$ [3]. Assuming the maximum energy from deuterium plasma in KSTAR, we used $3 \mathrm{MeV}$ mono energetic neutron source in which neutrons were emitted from toroidal region uniformly. We used the PHITS with JENDL4.0 database [4].

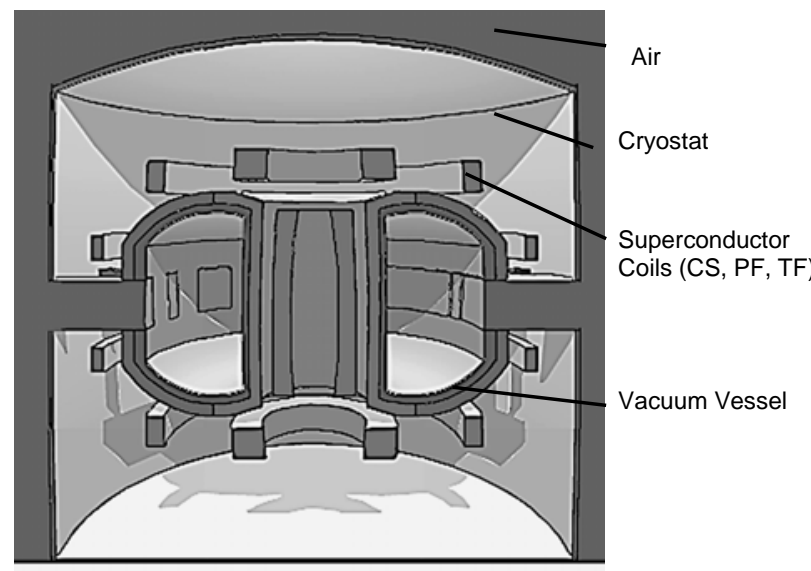

Figure 3. Monte-Carlo model of KSTAR and material specification.

\subsection{Shielding structure for APCCNS}

For collimation of incident neutron beam, we designed a collimator shown in Figure 4. The material of the collimator was polyethylene including ${ }^{10} \mathrm{~B}$ (density of $1.11 \mathrm{~g} / \mathrm{cm}^{3}$ ). Length of the collimator was 450 $\mathrm{mm}$ with a $20 \mathrm{~mm}$ diameter through-hole. We calculated neutron intensity after the collimator at J port of KSTAR using the calculation model. Then performance of the collimator was evaluated by intensity at peak energy of neutron i.e. passing through the hole without scattering and total intensity over the whole energy range. Figure 5 shows a dependence of the peak to total ratio on a thickness of the collimator. The peak to total ratio, i.e. a relative intensity of non-scattered neutron was almost saturated at the thickness of around $400 \mathrm{~mm}$ and the peak to total ratio of 0.7 would be achieved. To save a weight of the collimator, we constructed the collimator with a dimension of $\mathrm{H}: 400 \mathrm{~mm} \times \mathrm{W}: 400 \mathrm{~mm} \times \mathrm{L}: 450 \mathrm{~mm}$.

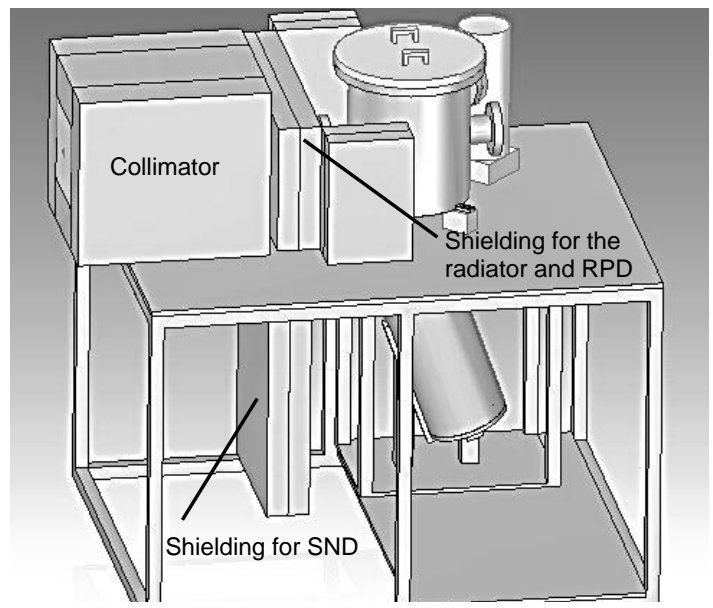

Figure 4. Geometry of the shielding structure of APCCNS.

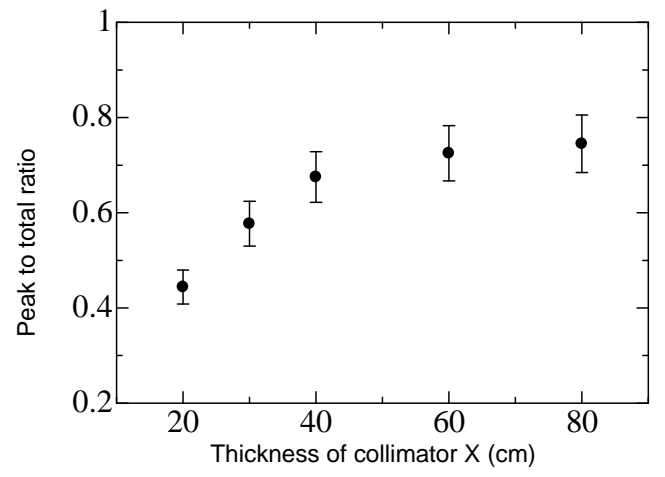

Figure 5. Dependence of the peak-total ratio on the thickness. The peak-total ratio is defined as a ratio between intensity at incident neutron energy i.e. non-scattered neutron and total intensity over the whole energy range including scattered neutron.

As a scattered neutron shielding, polyethylene including ${ }^{10} \mathrm{~B}$ was also adopted. In addition, further lead shielding was required to shield hard X-ray due to interaction of high energy electron with the first wall, the vacuum vessel and so on. We constructed a 
preliminary shielding structure with a lead thickness of 5 $\mathrm{cm}$ and $\mathrm{a}{ }^{10} \mathrm{~B}$ polyethylene thickness of $5 \mathrm{~cm}$ due to weight limits of the shielding structure. With a simplified model for calculation of scattered of the collimator, we estimated an attenuation effect defined as a ratio in intensities between neutrons passing through both the lead and the ${ }^{10} \mathrm{~B}$ polyethylene plates and incident neutrons. Here, neutron with energy of less than $0.05 \mathrm{MeV}$ was negligible because it was lower than detection threshold of the radiator and the scattered neutron detector. Figure 6 shows the dependence of the attenuation effect on incident neutron energy. For incident neutron energy of less than $0.2 \mathrm{MeV}$, scattered neutron was reduced by less than $10 \%$. For further evaluation, experimental testing of the shielding for scattered neutron and X-ray is required.

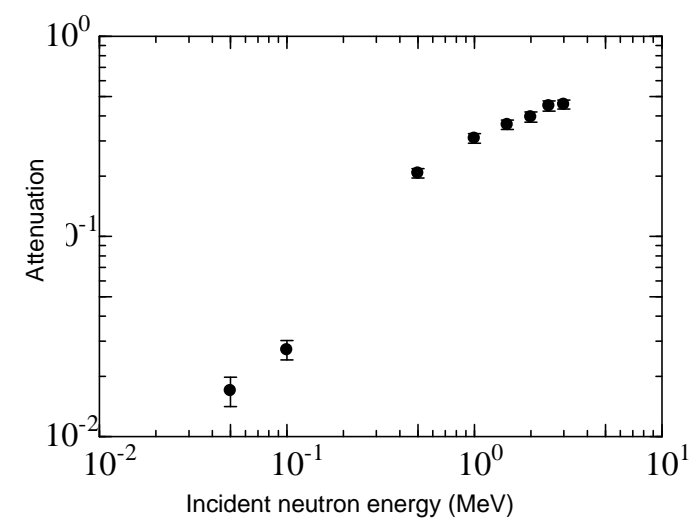

Figure 6. Attenuation effect by preliminary shielding structure with a lead thickness of $5 \mathrm{~cm}$ and a ${ }^{10} \mathrm{~B}$ polyethylene thickness of $5 \mathrm{~cm}$.

\section{Conclusion}

The prototype of APCCNS was installed on J port of KSTAR at NFRI toward deuterium plasma experiment. For neutronics design, the neutron transport calculation model of KSTAR was developed based on PHITS. The shielding structure including the collimator was designed and constructed. Shielding effect for high energy X-ray and neutron by the shielding structure will be evaluated experimentally. DD neutron measurement in deuterium plasma experiment at KSTAR will be started in 2012.

\section{Acknowledgements}

This work is performed with the support and under the auspices of the NIFS Collaboration Research program (NIFS12KOAH029), Japan-Korea Fusion Collaboration Program and JSPS-NRF-NSFC A3 Foresight Program in the field of Plasma Physics (NSFC: No.11261140328). The authors wish to thank Dr. Alan England and Dr. Y.S. Lee for the helpful comments to this paper.

\section{References}

[1] H. Tomita, H. Iwai, T. Iguchi, M. Isobe, J. Kawarabayashi and C. Konno, Development of neutron spectrometer toward deuterium plasma diagnostics in LHD, Rev. Sci. Instrum. 81 (2010), 10D309.

[2] K. Niita, N. Matsuda, Y. Iwamoto, H. Iwase, T. Sato, H. Nakashima, Y. Sakamoto and L. Sihver, PHITS: Particle and Heavy Ion Transport Code System, Version 2.23, JAEA-Data/Code 2010-022 (2010).

[3] Y.-K. Oh, W.C. Kim, K.R. Park et al., Commissioning and initial operation of KSTAR superconducting tokamak, Fus. Eng. Design 84 (2009), pp. 344-350.

[4] K. Shibata, O. Iwamoto, T. Nakagawa, N. Iwamoto, A. Ichihara, S. Kunieda, S. Chiba, K. Furutaka, N. Otuka, T. Ohsawa, T. Murata, H. Matsunobu, A. Zukeran, S. Kamada and J. Katakura, JENDL-4.0: A new library for nuclear science and engineering, J. Nucl. Sci. Technol. 48 (2011), pp.1-30. 